\title{
Two-Dimensional Photonic Crystal Resist Membrane Nanocavity Embedding Colloidal Dot-in-a-Rod Nanocrystals
}

\author{
Luigi Martiradonna, ${ }^{*,+, \ddagger}$ Luigi Carbone, ${ }^{\ddagger}$ Aniwat Tandaechanurat, ${ }^{\dagger}$ \\ Masatoshi Kitamura, ${ }^{\dagger}$ Satoshi Iwamoto, ${ }^{\dagger}$ Liberato Manna, ${ }^{\ddagger}$ Massimo De Vittorio, ${ }^{\ddagger}$ \\ Roberto Cingolani, $\neq$ and Yasuhiko Arakawa ${ }^{\dagger}$
}

\begin{abstract}
IIS, INQIE, RCAST, The University of Tokyo, 4-6-1 Komaba, Meguro-ku, 153-8904, Tokyo, Japan, and NNL, National Nanotechnology Laboratory of CNR-INFM, ISUFI, University of Salento, Via per Arnesano, I-73100, Lecce, Italy
\end{abstract}

Received October 5, 2007; Revised Manuscript Received December 5, 2007

\begin{abstract}
A novel technique for the fabrication of photonic crystal (PC) nanocavities coupled with colloidal nanocrystals is presented. A waveguiding resist membrane embedding highly emitting dot-in-a-rod nanocrystals was patterned through e-beam lithography and released through wet etching process. The proposed approach makes the PC structure independent of fabrication imperfections induced by etching steps. Microphotoluminescence spectra revealed degenerated resonant modes (Q-factor $\sim 700$ ) whose fabrication-induced spectral splitting is comparable to the full width at half-maximum of the peaks. Active nanocavities tunable from visible to infrared spectral range on GaAs or Si substrates can be easily implemented by this technique.
\end{abstract}

The development of photonic devices based on wet-chemically synthesized semiconductor nanocrystals (NCs) is nowadays one of the main research topics for nanophotonic devices and applications. The performances of colloidal NCs are in several aspects competitive with epitaxially grown quantum-emitters: they show broad excitation spectra and narrow emission bands also at room temperature, tunable optical gain and amplified spontaneous emission from the ultraviolet to the infrared spectral range, and a high photochemical stability, while requiring cheaper and higherthroughput growth facilities. ${ }^{1-3}$ Spectral shifting and blinking of the single-nanoparticle luminescence, which limited the NCs exploitation in single emitter applications or in fluorescence bio-labeling, are being deeply investigated ${ }^{4-6}$ and have been reduced by improved engineering of the surface chemistry. ${ }^{7,8}$ A wide range of optical devices based on colloidal quantum nanoemitters has been demonstrated, such as advanced organic light emitting diodes generating white luminescence, ${ }^{9-11}$ photovoltaic devices, ${ }^{12}$ infrared photodiodes, ${ }^{13}$ and distributed feedback lasers. ${ }^{14}$ Single photon emission from colloidal nanocrystals has also been recently obtained. ${ }^{15}$

* To whom correspondence should be addressed. Tel: +81-3-5452-6098

Fax: +81-3-5452-6247. E-mail: martirad@iis.u-tokyo.ac.jp.

The University of Tokyo.

$\doteqdot$ University of Salento.
The insertion of nanoemitters into photonic crystal (PC) cavities with localized optical modes having high quality factor $(\mathrm{Q})$ and small modal volume can be exploited to tailor both the spatial and spectral properties of the NCs radiation. Colloidal nanocrystals confined into two-dimensional PCs are promising candidates for the development of nextgeneration high performing optical devices such as single photon sources, ultralow threshold lasers, and nonlinear devices. ${ }^{16-18}$ At present, several works have been proposed in order to couple colloidal NCs to 2D-PC structures fabricated on $\mathrm{Si}$ or AlGaAs membrane layers. ${ }^{19-21}$ In these reported cases, high Q passive nanocavities were previously fabricated on solid substrates; $\mathrm{PbS}$ or PbSe colloidal nanocrystals emitting in the infrared spectral range were subsequently added by dipping or spin-coating techniques on top of the patterned layer. The coupling between the emitters and the quantum-confined optical environment was demonstrated by the presence of resonant peaks in the photoluminescence (PL) spectra collected on the fabricated devices. The pattern transfer on the rigid membrane layer typically requires high-resolution etching steps, which however could introduce fabrication imperfections in the structure (deviation of the hole radii, slightly angled sidewalls, high surface roughness after resist stripping), thus deteriorating the optical quality of the photonic crystal. Moreover, these techniques 
can be only applied to infrared-emitting colloidal nanocrystals, due to not negligible Si and AlGaAs absorption in the visible spectral range.

In this work, we propose the fabrication of semiconductor nanocrystal-based 2D-PC nanocavities by directly patterning and releasing a thin membrane of a functional material composed by NCs dispersed in a positive electronic resist (ZEP520-12). ${ }^{22}$ No pattern transfer from the resist to the underlying membrane layers is required, since the resist itself acts as the suspended waveguiding layer. Therefore the optical quality of the resonating structure is only determined by a single high-resolution lithographic step, whereas it is not affected by any etching process. Moreover, the absence of absorbing layers from the visible to the infrared region of the spectrum allows the use of NCs on a large spectral range, thus conferring a broader applicability to our technique.

Our approach can be applied to any organic or inorganic emitter provided that it can be dispersed in resist matrices. Nevertheless, in this prototype, we exploited a new type of semiconductor nanoemitters, which are dot-in-a-rod colloidal nanocrystals (DR-NCs) consisting of CdS rods nucleated at high temperatures around $\mathrm{CdSe}$ spherical seeds. ${ }^{23,24}$ These elongated core-shell nanocrystals remarkably show high quantum efficiency (up to $70-75 \%$ ), due to a high quantum yield coupled with a large extinction coefficient in the ultraviolet spectral range, the latter due to the presence of a thick CdS shell. Therefore their PL signal is easily detectable even at room temperature with no need of highly sensitive detection setups. ${ }^{25}$ Figure 1a shows a transmission electron microscopy (TEM) image of the as-grown samples, with a core diameter of $\sim 2.5 \mathrm{~nm}$ and a rod length of $\sim 16 \mathrm{~nm}$ (a sketch of the structure is reported in the inset), while in Figure $1 \mathrm{~b}$ their absorption (dashed line) and emission (solid line) spectra are reported. After growth, the DR-NCs were subsequently dispersed in a ZEP520-12 matrix ${ }^{22}$ with a molar concentration of $\sim 1 \times 10^{-6} \mathrm{~mol} / \mathrm{L}$. As previously reported, ${ }^{26}$ blends composed by colloidal nanocrystals dispersed in electronic resists can be easily patterned by means of traditional electron-beam lithography, since the presence of semiconductor clusters in the matrix does not affect the sensitivity of the polymeric host significantly. Optical microscopy and scanning electron microscopy (SEM) analyses on thin films of the compound material (not shown) assessed the absence of aggregates induced by phase segregation between the polymer and the semiconductor nanoclusters. Further characterization performed with a Woollam EC-400 Ellipsometer showed a refractive index $n$ $=1.575$ at $\lambda=600 \mathrm{~nm}$.

In order to design a suitable 2D-PC cavity, threedimensional (3D) finite-difference time-domain (FDTD) calculations were carried out. ${ }^{27}$ Due to the low index contrast between the membrane and the surrounding air cladding, the simulation of 2D-PC cavities (such as H1 or L3 defect cavities $)^{28-29}$ led to localized modes with a Q-factor of only few hundreds. ${ }^{30}$ In order to increase the mode confinement, an approach similar to the graded square lattice proposed by Srinivasan et al. ${ }^{31}$ was followed. Starting from a regular
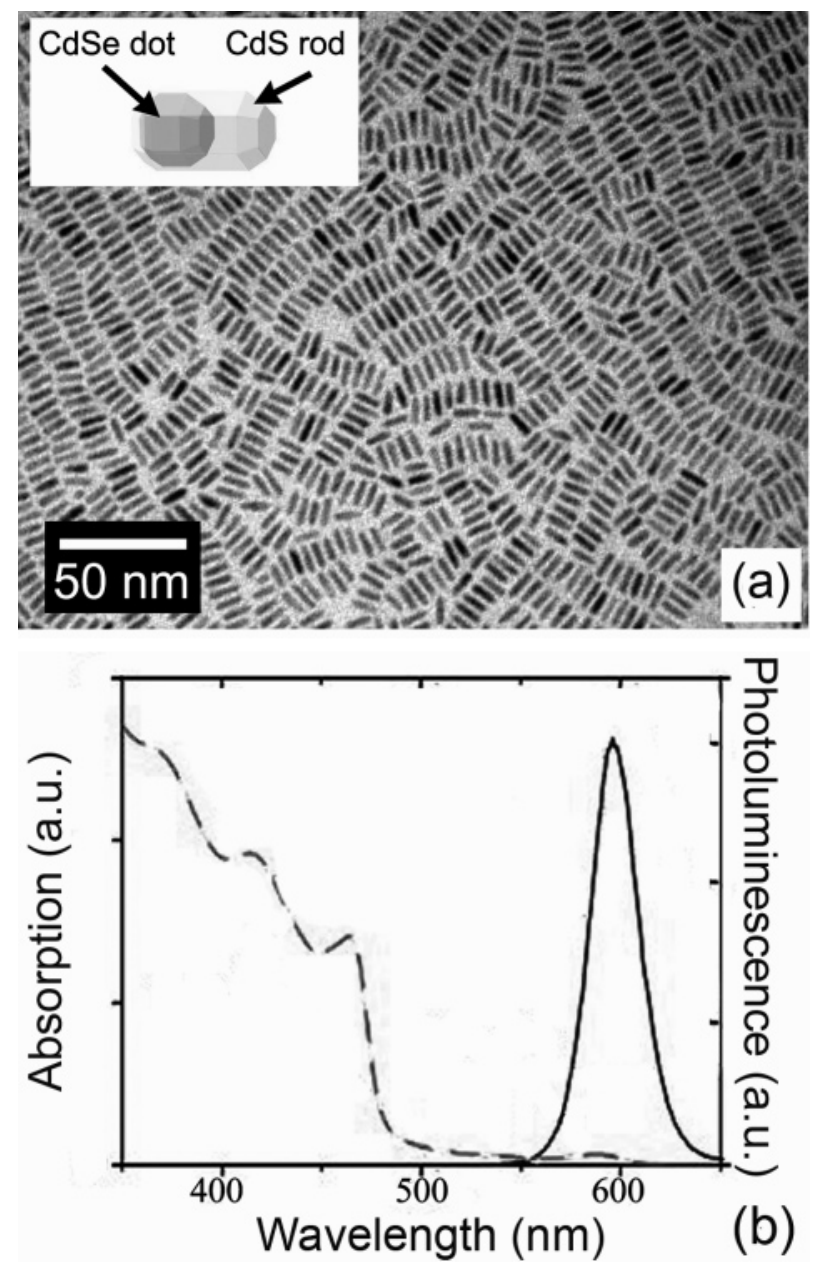

Figure 1. (a) Transmission electron microscope image of the colloidal dot-in-a-rod nanocrystals exploited in this work (inset: sketch of the CdSe dot/CdS rod structure). (b) Emission (solid line) and absorption (dashed line) spectra of the dot-in-a-rod nanocrystals.

square lattice PC structure, we chose resonant modes whose main electric field distribution was found to be odd-symmetric with respect to mirror planes normal to their dominant Fourier components. This leads to the suppression of the resonant mode energy fraction coupled with out-of-plane radiating fields. At the same time, a graded reduction of the air hole radii from the external rings to the inner ones following a parabolic law was applied, in order to avoid the coupling of the localized mode with in-plane leaky modes in the momentum space. ${ }^{31}$ Additionally, we exploited a quadratic grading of the holes by applying the same parabolic law in both $x$ and $y$ directions. In this case the final defect structure preserves a $C_{4 v}$ symmetry and therefore degenerated localized modes with orthogonal polarization can be predicted. Figure 2a reports a sketch of the implemented 2DPC pattern, with black lines at the margins indicating the parabolic law used along $x$ and $y$ to decrease the ratio of holes radius to lattice constant (r/a) from 0.4 to 0.2 . Figure 2, panels $\mathrm{b}$ and $\mathrm{c}$, show the spatial distribution of the electric field intensity of two degenerated modes localized at a normalized frequency equal to $a / \lambda=0.404$; the two modes are orthogonally polarized. Inset in Figure $2 \mathrm{~b}$ also shows the electric field amplitude distribution of the first localized 

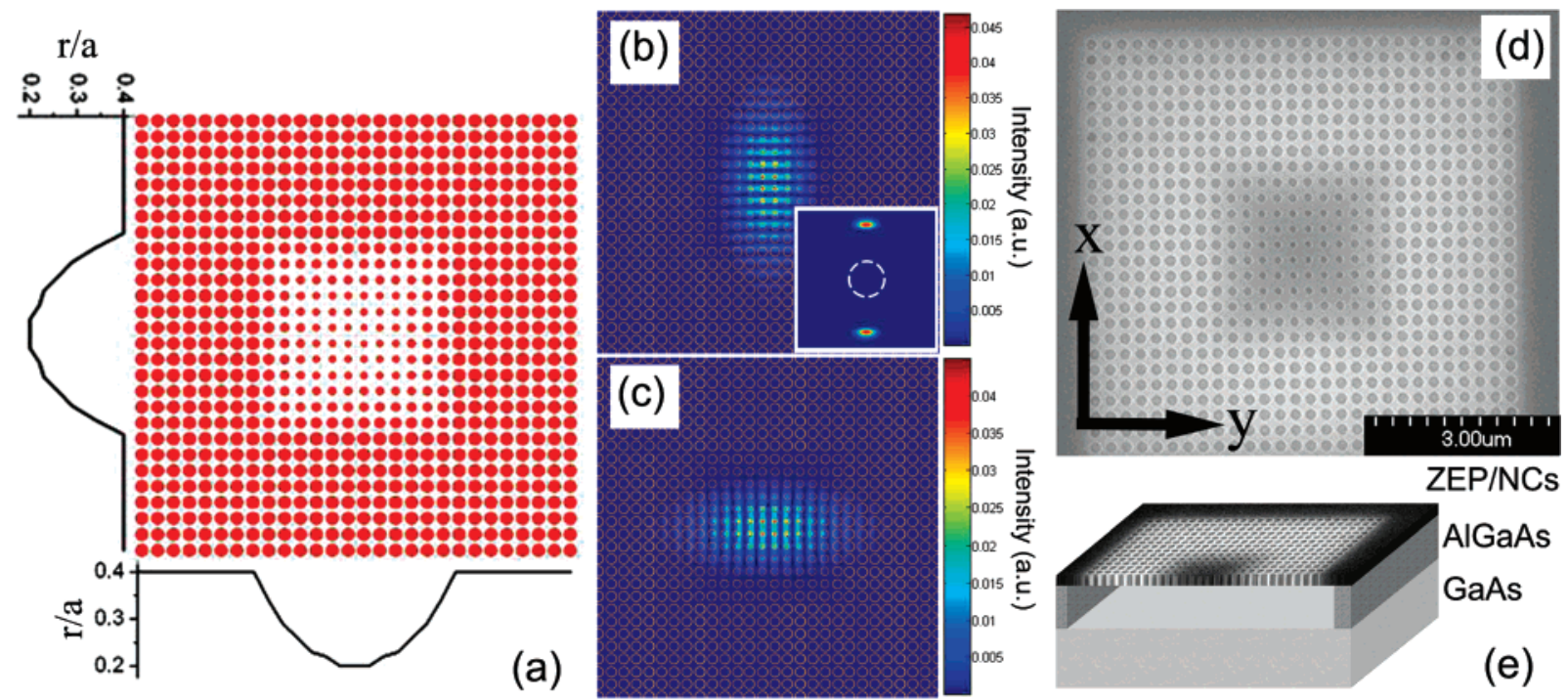

Figure 2. (a) Sketch of the photonic crystal nanocavity in square lattice with modulated air hole radii along $x$ and $y$. (b,c) FDTD calculated intensity distributions of two fundamental modes, degenerated at $a / \lambda=0.404$. The inset in (b) shows the Fourier transformed dominant electric field component. The field fraction inside the light cone (dashed circle in the figure) is strongly reduced, thus assessing the significant suppression of vertical radiation. (d) Scanning electron microscope image of the patterned nanocavity; (e) sketch of the final membrane structure, obtained after wet etching of the $\mathrm{Al}_{0.7} \mathrm{Ga}_{0.3} \mathrm{As}$ sacrificial layer.

mode in the momentum space. The field components lying inside the light-cone line (represented by the white dashed line) are strongly suppressed due to the odd symmetry of the mode: coupling with out-of-plane leaky modes is therefore reduced. Furthermore, the overlap between the electric field amplitude and the guiding modes in the inplane direction (not shown in the inset) is also reduced due to the holes radii grading, thus suppressing the photon leakage into the lateral direction. The Q-factor calculated from the modal energy decay rate was equal to $\sim 5700$, with a modal volume equal to $\sim 1.8(\lambda / n) .^{3}$

For the practical realization of the device, a $1 \mu \mathrm{m}$ thick layer of $\mathrm{Al}_{0.7} \mathrm{Ga}_{0.3} \mathrm{As}$ was epitaxially grown by metal-organic chemical vapor deposition (MOCVD) on GaAs substrate and a $320 \mathrm{~nm}$ thick layer of ZEP/NCs was spin coated on top of it. Several graded square lattice patterns with different lattice constants (ranging from $a=220 \mathrm{~nm}$ to $a=258 \mathrm{~nm}$ ) were then transferred on the layer by an EBL process. ${ }^{32}$ Figure $2 \mathrm{~d}$ shows an in-plane SEM image of the patterned structure after development. In order to remove the underlying sacrificial layer, the sample was then dipped in a $\mathrm{HF}: \mathrm{H}_{2} \mathrm{O}$ etching solution at room temperature. It was then rinsed with isopropilic alcohol with the aim to decrease the surface tension of the solvent during its evaporation in air. A sketch of the final structure after wet-etching is shown in Figure 2e.

In order to verify the effectiveness of the fabrication process, micro-photoluminescence measurements at room temperature were performed on the nanocavities. The sample was mounted on a micro-translational vertical holder and excited by a $\mathrm{CW}$ diode laser emitting at $\lambda=405 \mathrm{~nm}$ (excitation power: $500 \mathrm{nW}$ ), through a $50 \times$ objective lens. The emission was collected through the same objective lens and filtered by a polarizer and a pinhole in order to reduce further the collection area to the central graded lattice region of the nanocavity. The signal was detected by means of a monochromator equipped with a liquid $\mathrm{N}_{2}$ cooled CCD. All the measurements were performed in air, at room temperature.

Figure 3a shows a typical spectrum collected from the suspended resist cavity. A sharp peak, with a Q-factor $\sim 700$, is present in the emission spectrum, due to the resonance effect of the 2D-PC pattern. Besides the fundamental mode, several other modes have also been detected from the cavity (as shown in Figure 3a). According to simulations, all Q-factors of higher modes are lower than the fundamental mode, both due to the even symmetry in the distribution of the main electric field component (which decreases the vertical confinement) and to a higher overlap with in-plane leaky modes in the momentum space.

The polarization dependence of the fundamental mode was also studied, and nearly degenerated modes with an average spectral splitting $\sim 1 \mathrm{~nm}$ (averaged on more than 200 samples tested, with a standard deviation of $0.42 \mathrm{~nm}$ ), comparable to their fwhm, were detected. These can be seen in Figure $3 \mathrm{~b}$, which shows three different spectra collected from the same cavity at different polarization angles (the spectra have been shifted along the vertical axes to improve the clarity of the graph). The minimum measured spectral splitting was as low as $0.4 \mathrm{~nm}$. Furthermore, these modes showed orthogonal polarization (see the polar diagram of the two modes in Figure 3c), as expected from the 3D-FDTD calculations.

Remarkably, the fabrication approach showed high accuracy, since different tested samples fabricated with the same lithographic conditions showed very low standard deviations in the resonant peak position (standard deviation $\sim 0.34 \mathrm{~nm}$ ) and in the fwhm $(\sim 0.055 \mathrm{~nm}$, below the resolution limit of our detection setup). Reproducibility near to $100 \%$ has been verified, with deviations of different samples from the 

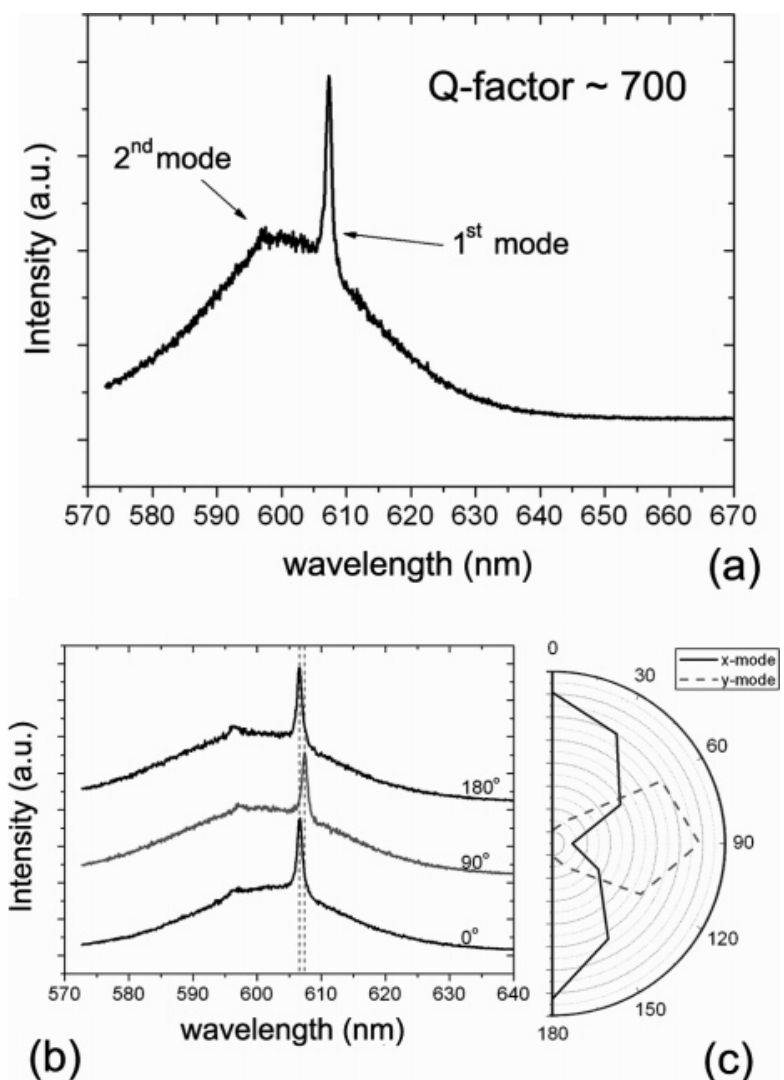

Figure 3. Micro-PL analysis of the nanocavity at room temperature. (a) Emission detected from the nanocavity $\left(\lambda_{\mathrm{exc}}=405\right.$ $\mathrm{nm})$, showing a sharp resonant peak corresponding to the fundamental mode $(Q \sim 700)$ and a smaller resonant peak corresponding to the second-order mode. (b) PL spectra collected through a polarizer from the same nanocavity, at different polarization angles. Two degenerated modes with a small spectral splitting (0.8 $\mathrm{nm}$ ) are detected; (c) the polar diagram shows their orthogonal polarization.

expected behavior mainly due to the presence of local impurities in the spin-coated layer or to variations in the polymeric layer thickness.

The good match between the optical measurements and the theoretical predictions demonstrates the effectiveness of this new simple two-step process (EBL pattern definition followed by wet etching of the sacrificial layer), which enables the fabrication of colloidal nanocrystal-based active photonic devices without recurring to high-resolution dry etching steps. The difference between the experimental Q-factor for the fundamental mode and the simulated one is less than 1 order of magnitude. ${ }^{33}$ At present, comparable experimental Q-factor values reported in literature for 2D-PCs cavities based on colloidal nanocrystals typically show degradation from the simulated Q-factor by 2 orders of magnitude, ${ }^{21}$ due to a higher number of imperfections in the fabrication steps. In addition, the robustness of our process is also assessed by the high accuracy and reproducibility of the main figures of merit of the resonant structure and by the small average splitting between the degenerated $x$ and $y$ polarized modes. This low value shows that the symmetry of the quadratically graded square lattice structure is well preserved in final structure.
It is worth noting that this fabrication technique has been demonstrated on colloidal nanocrystals emitting in the visible spectral range. The use of air claddings and the intrinsic transparency of the resist matrix in both the visible and the infrared spectral range extends the applicability of NC based 2D-PC cavities from the infrared emitting $\mathrm{PbS}$ or $\mathrm{PbSe} \mathrm{NCs}$ to several types of core-shell NCs emitting in the visible spectral range and, therefore, also to the new high-performing dot-in-a-rod colloidal nanocrystals as reported here. Interestingly, despite the process has been demonstrated on GaAs substrates with AlGaAs sacrificial layers, it can be transferred straightforwardly to $\mathrm{Si}$ substrates with $\mathrm{SiO}_{2}$ sacrificial layers, so that NC-based optical sources fully integrated with Silicon technology can be fabricated. Additionally, the intrinsic elasticity of the polymeric membranes could allow the development of pressure sensitive tunable emitters based on the modification of the nanocavity spectral response when deforming forces are applied. An additional future development of this approach would lie in the localization of single $\mathrm{NC}$ emitters in a nanocavity (if the NCs concentration in the polymeric blend is further decreased), which could allow the fabrication of ultralow-threshold lasers or efficient singlephoton sources.

In conclusion, a new approach for the fabrication of 2D-PC cavities based on colloidal nanocrystals has been proposed. The photonic cavity is directly fabricated on a resist layer that embeds the semiconductor nanoemitters and it is then suspended by means of a wet etching process. High Q-factor degenerated localized modes (Q 700) have been detected, with an average spectral splitting as low as the fwhm of the modes, thus assessing the good preservation of the pattern symmetry from the designed cavity to the fabricated structure. This new strategy for the fabrication of high-performing NCs-based devices is clearly promising for the development of all-optical circuits that can be fully integrated with traditional Silicon technology.

Acknowledgment. This work was developed in the Joint Research collaboration program between IIS, Japan, and NNL, Italy and the Specially Appointed Funds for Promoting Science and Technology. The authors gratefully acknowledge T. Nakaoka, M. Nomura, P. P. Pompa, and G. Morello for useful discussions. The expert technical help of B. Antonazzo, S. Ishida, and M. Nishioka is also acknowledged.

\section{References}

(1) Alivisatos, A. P. Science 1996, 271, 933.

(2) Manna, L.; Scher, E. C.; Alivisatos, A. P. J. Am. Chem. Soc. 2000, $122,12700$.

(3) Klimov, V. I.; Mikhailovsky, A. A.; Xu, S.; Malko, A.; Hollingsworth, J. A.; Leatherdale, C. A.; Eisler, H.-J.; Bawendi, M. G. Science 2000, 290, 314.

(4) Nirmal, M.; Dabbousi, B. O.; Bawendi, M. G.; Macklin, J. J.; Trautman, J. K.; Harris, T. D.; Brus, L. E. Nature 1996, 383, 802

(5) Fisher, B. R.; Eisler, H.-J.; Stott, N. E.; Bawendi, M. G. J. Phys. Chem. B 2004, 108, 143.

(6) Briebricher, A.; Sauer, M.; Tinnefeld, P. J. Phys. Chem. B 2006, 110, 5174.

(7) Parak, W. J.; Gerion, D.; Pellegrino, T.; Zanchet, D.; Micheel, C; Williams, S. C.; Boudreau, R.; Le Gros, M. A.; Larabell, C. A.; Alivisatos, A. P. Nanotechnology 2003, 14, R15.

(8) Hohng, S.; Ha, T. J. Am. Chem. Soc. 2004, 126, 1324. 
(9) Sheats, J. R.; Antoniadis, H.; Hueschen, M.; Leonard, W.; Miller, J.; Moon, R.; Roitman, D.; Stocking, A. Science 1996, 273, 884.

(10) Colvin, V. L.; Schlamp, M. C.; Alivisatos, A. P. Nature 1994, 370 , 354 .

(11) Jialong, Z.; Bardecker, J. A.; Munro, A. M.; Liu, M. S.; Yuhua, N.; I-Kang, D.; Jingdong, L.; Baoguan, C.; Jen, A. K.-Y.; Ginger, D. S. Nano Lett. 2006, 6 (3), 463.

(12) Huynh, W. U.; Dittmer, J. J.; Alivisatos, A. P. Science 2002, 295, 2425.

(13) Cui, D.; Jian, X.; Sheng-Youg, X.; Paradee, G.; Lewis, B. A.; Gerhold, M. D. IEEE Trans. Nanotechnol. 2006, 5 (4), 362.

(14) Sundar, V. C.; Eisler, H.-J.; Deng, T.; Chan, Y.; Thomas, E. L.; Bawendi, M. G. Adv. Mater. 2004, 16, 2137.

(15) Brookmann, X.; Giacobino, E.; Dahan, M.; Hermier, J. P. Appl. Phys Lett. 2004, 85, 712.

(16) Englund, D.; Fattal, D.; Waks, E.; Solomon, G.; Zhang, B.; Nakaoka, T.; Arakawa, Y.; Yamamoto, Y.; Vučković, J. Phys. Rev. Lett. 2005, 95, 013904.

(17) Yablonovitch, E. J. Opt. Soc. Am. B 1993, 10, 293.

(18) Soljačić, M.; Joannopoulos, J. D. Nat. Mater. 2004, 3, 221.

(19) Fushman, I.; Englund, D.; Vuckovic, J. Appl. Phys. Lett. 2005, 87, 241102 .

(20) Bose, R.; Yang, X.; Chatterjee, R.; Gao, J.; Wong, C. W. Appl. Phys. Lett. 2007, 90, 111117.

(21) Wu, Z.; Mi, Z.; Bhattacharya, P.; Zhu, T.; Xu, J. Appl. Phys. Lett. 2007, 90, 171105 .

(22) ZEP520-12 electronic resist diluted in o-dichlorobenzene, from ZEON Corporation (www.zeon.co.jp).

(23) Carbone, L.; Nobile, C.; De Giorgi, M.; Della Sala, F.; Morello, G.; Pompa, P. P.; Hytch, M.; Snoeck, E.; Fiore, A.; Franchini, I. R.; Nadasan, M.; Silvestre, A. F.; Chiodo, L.; Kudera, S.; Cingolani, R.; Krahne, R.; Manna, L. Nano Lett. 2007, 7 (10), 2942.

(24) Talapin, D. V.; Nelson, J. H.; Shevchenko, E. V.; Aloni, S.; Sadtler, B.; Alivisatos A. P. Nano Lett. 2007, 7 (10), 2951.

(25) The seeded growth approach allows to grow core/shell nanorod structures at high temperatures $\left(>350^{\circ} \mathrm{C}\right)$, and this leads to highly monodisperse elongated structures with few crystallographic defects.
The elongated shell strongly increases the absorption cross-section of the nanocrystals in the blue - UV region with respect to the absorption of spherical core-shell nanocrystals with comparable quantum yield, and therefore a brighter emission is expected. The Stokes shift between the first excitonic peak and the DR-NC emission peak (the latter centered at $\sim 600 \mathrm{~nm}$ with a full-width-at-halfmaximum (FWHM) of $24 \mathrm{~nm}$ ) is only slightly affected by the rod length, therefore confirming that the radiative recombination process is mainly localized in the core (i.e., seed) region.

(26) Martiradonna, L.; Stomeo, T.; Carbone, L.; Morello, G.; Salhi, A.; De Giorgi, M.; Cingolani, R.; De Vittorio, M. Phys. Status Solidi B 2006, 15, 3972.

(27) Taflove, A.; Hagness, S. C. The finite-difference time-domain method. Computational Electrodynamics; Artech House: London, 2000.

(28) Shirane, M.; Kono, S.; Ushida, J.; Onkouchi, S.; Ikeda, N.; Sugimoto, Y.; Tomita, A. J. Appl. Phys. 2007, 101, 073107.

(29) Akahane, Y.; Asano, T.; Song, B.-S.; Noda, S. Nature 2003, 425, 944.

(30) This low $Q$ value is limited by large losses in both out-of-plane and in-plane directions because photonic band-gap exists in only relatively high frequency regions and it is at best very narrow.

(31) Srinivasan, K.; Painter, O. Opt. Express 2002, 10, 670.

(32) The electron beam lithography process was carried out on a JEOL JBX-6000 lithography system (acceleration voltage $=50 \mathrm{KV}$, beam current $=120 \mathrm{pA}$ ), with modulated dose along the pattern in order to compensate the proximity effect between neighboring holes. The sample was then developed in ZED-N50 solvent and rinsed in ZMD-B (both from ZEON Corporation).

(33) Slight deviations (in the range of the resolution limit of the lithographic process) of the fabricated inner holes radii from the expected values can explain the reduction of the experimental $Q$-factor from the simulated one. Further improvements in the control of the lithographic exposure parameters will predictably lead to a better match between theoretical and experimental performances.

NL0725751 